\title{
Editorial
}

Int Neurourol J 2013;17:1

http://dx.doi.org/10.5213/inj.2013.17.1.1

pISSN 2093-4777 · eISSN 2093-6931

\section{Lab-on-a-chip for Urology}

\author{
Khae Hawn Kim \\ Associate Editor \\ Department of Urology, Gachon University Gil Medical Center, Incheon, Korea \\ E-mail: kimcho99@gilhospital.com
}

In an effort to make a diagnosis of diseases, to assess the treatment outcomes and to evaluate the prognosis, many investigators are currently dedicating themselves to identifying disease markers more easily through a minimally-invasive method using human samples. This has eventually improved the results of critical analysis of individual steps of the various methods. Nevertheless, much scientific attention has been focused on the methodology rather than on the development of devices. Still, samples or specimens are prepared in a test tube or a petri dish although they can be processed in large volumes irrespective of the technique. With the exceptions of some biomarkers and micromanipulation, there are no technological advancements leading to the widespread use in the field of urology. However, more so than changes in a procedure or methodology as technical advancements have had a great effect on the clinical application. In this issue of International Neurourology Journal, Lee [1] introduces the concept of the Lab-on-a-chip technology, which is based on the microfluidic system. As the state-of-theart technology, the "Lab-on-a-chip" is based on the miniaturization of laboratory apparatus, and it is a technology that has been gaining increasing interest. It is not just a methodology, and it focuses on the development of technology. In other words, it aims to construct a totally-integrated, disposable microfluidic system that is operated by a single mode. Moreover, it generates pressure that can be applied on-site with an enhanced portability and disposability and that can be operated more simply and rapidly for making medical, surgical and clinical diagnoses. Compared with the application of the lab-on-a-chip in other clinical specialties, the application of this technology in the field of urology remains unnoticeable, although it is presumed to be applicable for diagnosing urinary tract infection or for performing in vitro fertilization [2,3]. Of course, it is evident that there are many obstacles in integrating and making a clinical application of it. It would therefore be mandatory to develop reliable methods for directing flow through a network of channels. In the field of neurourology, uro-oncology, and nephrology, urine is treated as valuable human samples. Many investigators have great interest in identifying disease makers with the use of human urine samples and in enabling the clinical application of such methods. To date, however, no studies have reported the successful results. This leads to the speculation that we would achieve great things if we make an effort to understand the current state-of-the-art system and then to apply it to the diagnosis of urologic diseases. Thus, it will be established as a novel innovative technology. Keeping pace with the current trends, the review article of this issue will provide a high-level understanding of micro- and nanotechnologies, novel research areas that are making a rapid progress.

\section{REFERENCES}

1. Lee NY. Recent progress in lab-on-a-chip technology and Its potential application to clinical diagnoses. Int Neurourol J 2013;17:2-10.

2. Mach KE, Wong PK, Liao JC. Biosensor diagnosis of urinary tract infections: a path to better treatment? Trends Pharmacol Sci 2011; 32:330-6.

3. Suh R, Takayama S, Smith GD. Microfluidic applications for andrology. J Androl 2005;26:664-70.

This is an Open Access article distributed under the terms of the Creative Commons Attribution Non-Commercial License (http://creativecommons.org/licenses/by-nc/3.0/) which permits unrestricted non-commercial use, distribution, and reproduction in any medium, provided the original work is properly cited. 